\title{
THE SIMILARITY ORBIT OF A NORMAL OPERATOR
}

BY

\section{A. FIALKOW}

ABSTRACT. If $N$ is a bounded normal operator on a separable Hilbert space $H$, let $S(N)$ denote the similarity orbit of $N$ in $L(H)$ and let $S_{k}(N)$ denote the set of all compact perturbations of elements of $S(N)$. It is proved that $S(N)\left(S_{K}(N)\right)$ is norm closed in $L(H)$ if and only if the spectrum (essential spectrum) of $N$ is finite. If the essential spectrum of $N$ is infinite and $M$ is a normal operator whose spectrum is connected and contains that of $N$, then $M$ is in the closure of $S(N)$. If the spectrum of $N$ is connected, this result characterizes the normal elements of the closure of $S(N)$. A normal operator is similar to a nonquasidiagonal operator if and only if its essential spectrum contains more than two points.

1. Introduction. Let $H$ denote a fixed, separable, infinite dimensional, complex Hilbert space and let $L(H)$ denote the algebra of all bounded linear operators on $H$. If $N$ is a normal operator in $L(H)$, define the following subsets of $L(H)$ : $S(N)=\left\{X N X^{-1}: X\right.$ is invertible in $\left.L(H)\right\}$; $U(N)=\left\{U N U^{*}: U\right.$ is unitary $\}$;

$S_{K}(N)=\left\{X N X^{-1}+C: X\right.$ is invertible and $C$ is compact in $\left.L(H)\right\}$; $U_{K}(N)=\left\{U N U^{*}+C: U\right.$ is unitary and $C$ is compact $\}$. $S(N)$ is the similarity orbit of $N$ and $U(N)$ is the unitary orbit. Two consequences of Theorem A (below) are that the norm closure of $U(N)$ in $L(H)$ is contained in $U_{K}(N)$ and that $U_{K}(N)$ is closed in $L(H)$, i.e. $U(N)^{-} \subset U_{K}(N)=U_{K}(N)^{-}$. One purpose of this note is to examine the closure properties of $S(N)$ and $S_{K}(N)$. Since similarity is a weaker relation than unitary equivalence, we cannot expect a close analogue to the unitary case.

Our basic result is that $S(N)$ is closed if and only if $\sigma(N)$ is finite and $S_{K}(N)$ is closed if and only if $E(N)$ is finite (see below for notation). We prove that if $E(N)$ is infinite, and $M$ is a normal operator whose spectrum is connected and contains that of $N$, then $M$ is in $S(N)^{-}$; in particular $S(N)^{-} \not \subset S_{K}(N)$. As a consequence, we prove that if $\sigma(N)$ is connected, then a normal operator $M$ is in $S(N)^{-}$if and only if $\sigma(M)$ is connected and $\sigma(N) \subset \sigma(M)$. It is known that two normal operators that are similar (or even quasi-similar) are unitarily equivalent [9]. In contrast to this theorem, our results imply that if $N$ is a normal operator, then each normal operator in $S(N)^{-}$is in $U(N)^{-}$if and only if $E(N)$ is finite. 
In $\S 4$ we prove that a normal operator is similar to a nonquasidiagonal operator if and only if $E(N)$ contains more than two points.

We conclude this section with some notation. All Hilbert spaces in this note are separable. If $T$ is in $L(H)$ we denote the spectrum of $T$ by $\sigma(T)=$ $\{z: T-z$ is not invertible in $L(H)\}$; the resolvent of $T$ is $\rho(T)=\mathbf{C}-\sigma(T)$; the approximate point spectrum of $T$ is $\pi(T)=\{z: T-z$ is not bounded below $\}$. Let $C$ denote the ideal of all compact operators in $L(H)$ and let $\widetilde{T}$ denote the image of $T$ under the canonical homomorphism of $L(H)$ onto the Calkin algebra $L(H) / C$. The essential spectrum of $T$ is $E(T)=\{z: \widetilde{T}-z$ is not invertible in $L(H) / C\}=\{z: T-z$ is not a Fredholm operator $\}$ (see [3]). Note that if $T$ is in $S(N)^{-}$, then $\sigma(N) \subset \sigma(T)(E(N) \subset E(T))$ since the set of all invertible (Fredholm) operators is open in $L(H)$ [3]. A (diagonable) normal operator with finite spectrum $\left\{z_{1}, \ldots, z_{n}\right\}$ is denoted by $\operatorname{diag}\left(z_{i}\right)$. Following [6] we say that $N$ is an essential normal operator if $N$ is normal and $\sigma(N)=E(N)$. We state for reference the following results which will be used repeatedly in the sequel.

THEOREM A [1]. If $N$ is a normal operator in $L(H)$ and $M$ is an operator on $H$ such that $M^{*} M-M M^{*}$ is compact, $E(N)=E(M)$, and $\operatorname{index}(M-z)=0$ for each $z$ not in $E(M)$, then $M$ is unitarily equivalent to a compact perturbation of $N$.

THEOREM B [6, LEMMA 4]. If $N$ and $M$ are essential normal operators in $L(H)$ with the same spectrum, and if $\epsilon>0$, then there exist a unitary operator $U$ and a compact operator $K$ such that $N=U^{*} M U+K$ and $\|K\|<\epsilon$.

2. Operators with closed similarity orbits. In this section we prove that $S(N)$ is closed in $L(H)$ if and only if the spectrum of $N$ is finite. We begin with a well-known folk lemma.

Lemma 2.1. Let $z_{1}, \ldots, z_{n}$ be distinct complex numbers. Let $H_{1}, \ldots, H_{n}$ be Hilbert spaces and let $T$ be an operator on $H_{1} \oplus \ldots \oplus H_{n}$ with upper triangular operator matrix $\left(T_{i j}\right)$ such that $T_{i i}=z_{i}$, for $1 \leqslant i \leqslant n$. Then $T$ is similar to $\operatorname{diag}\left(z_{i}\right)$.

Proof. The proof is trivial if $n=1$. We assume the result is true for $n=k$ and prove the case $n=k+1$. If $H_{0}=H_{2} \oplus \ldots \oplus H_{k+1}$, then as an operator on $H_{1} \oplus H_{0}, T$ has a matrix representation of the form

$$
\left(\begin{array}{ll}
z_{1} & A \\
0 & B
\end{array}\right) \text {. }
$$

By induction, there is an invertible operator $S$ on $H_{0}$ such that $S B S^{-1}=$ 
$\operatorname{diag}_{2 \leqslant i \leqslant k+1}\left(z_{i}\right)$. Then $B-z_{1}$ is invertible and we set $X=-A\left(B-z_{1}\right)^{-1}$. If $R$ is the matrix on $H_{1} \oplus H_{0}$ of the form $\left(\begin{array}{ll}1 & X \\ 0 & S\end{array}\right)$, then a calculation shows that $R T R^{-1}=\operatorname{diag}\left(z_{i}\right)$.

LeMma 2.2. Let $z_{1}, \ldots, z_{n}$ be distinct complex numbers and let $D$ be a normal operator on $H$ with $\sigma(D)=\left\{z_{1}, \ldots, z_{n}\right\}$. If $\left\{S_{k}\right\}$ is a sequence of invertible operators on $H$ such that $\lim \left\|S_{k} D S_{k}^{-1}-T\right\|=0$, then $T$ is similar to $D$.

Proof. Let $p(z)=\left(z-z_{1}\right) \ldots\left(z-z_{n}\right)$. Since for each $k$ we have $p\left(S_{k} D S_{k}^{-1}\right)=S_{k} p(D) S_{k}^{-1}=0$, then clearly $p(T)=0$. If $q$ is a polynomial such that $q(T)=0$, then $q(\sigma(T))=\{0\}$, and since $\sigma(D) \subset \sigma(T)$, then $q(\sigma(D))=\{0\}$. Therefore $p$ divides $q$ and is thus the minimal polynomial of $T$. It follows from [5, Theorem 1] that there is an orthogonal family of subspaces of $H,\left\{H_{1}, \ldots, H_{n}\right\}$, such that $H=H_{1} \oplus \ldots \oplus H_{n}$ and such that with respect to this decomposition $T$ has an upper triangular operator matrix $\left(T_{i j}\right)$ with each $T_{i i}=z_{i}$. Lemma 2.1 implies that $T$ is similar to a diagonable operator $N$ such that $\sigma(N)=\sigma(D)$. To complete the proof it suffices to show that $\operatorname{dim} \operatorname{ker} N-z_{i}=\operatorname{dim} \operatorname{ker} D-z_{i}$ for each $i$.

If $1 \leqslant i \leqslant n$, there is a polynomial $q_{i}$ such that $q_{i}(D)$ is the projection whose kernel is $\operatorname{ker} D-z_{i}$ and such that $q_{i}(N)$ is the projection whose kernel is $\operatorname{ker} N-z_{i}$. Then $\operatorname{dim} \operatorname{ker} N-z_{i}=\operatorname{dim} \operatorname{ker} q_{i}(N)=\operatorname{dim} \operatorname{ker} q_{i}(T)$, and for each $k$, $\operatorname{dim} \operatorname{ker} D-z_{i}=\operatorname{dim} \operatorname{ker} q_{i}(D)=\operatorname{dim} \operatorname{ker} S_{k} q_{i}(D) S_{k}^{-1}$. Suppose that $\operatorname{dim} \operatorname{ker} q_{i}(D)>\operatorname{dim} \operatorname{ker} q_{i}(T)$. Let $k$ be an integer such that $\| S_{k} q_{i}(D) S_{k}^{-1}-$ $q_{i}(T) \|<1$ and let $x$ be a unit vector in the intersection of the kernel of $S_{k} q_{i}(D) S_{k}^{-1}$ and the initial space of $q_{i}(T)$. Then we have

$$
1>\left\|S_{k} q_{i}(D) S_{k}^{-1} x-q_{i}(T) x\right\|=\left\|q_{i}(T) x\right\| .
$$

Since each $S_{j} q_{i}(D) S_{j}^{-1}$ is an idempotent, it follows that $q_{i}(T)$ is an idempotent and is therefore bounded below by 1 on its initial space. This contradiction implies that $\operatorname{dim} \operatorname{ker} D-z_{i} \leqslant \operatorname{dim} \operatorname{ker} N-z_{i}$, and the reverse inequality is proved similarly.

LEMMA 2.3. Let $N$ be a normal operator on $H$ whose spectrum is not finite. There exist a sequence $\left\{U_{n}\right\}$ of unitary operators on $H$, and a normal operator $N_{1}$ that is not similar to $N$, such that $\lim \left\|U_{n} N U_{n}^{*}-N_{1}\right\|=0$.

Proof. Let $z$ be a limit point of $\sigma(N)$. If $z$ is not an eigenvalue of $N$ with infinite multiplicity, let $N_{1}=N \oplus z$, acting on $H \oplus H ; N_{1}$ is not similar to $N$. Since $z$ is in $E(N)$, Theorem 1 of [11] implies that $N_{1}$ is in $U(N)^{-}$. If $z$ is an ei- 
genvalue of $N$ with infinite multiplicity, there is a nontrivial orthogonal decomposition $H=H_{1} \oplus H_{2}$, with respect to which $N=N_{1} \oplus z$, and such that $z$ is not an eigenvalue of $N_{1}$. Since $z$ is a limit point of $\sigma(N), z$ is in $E\left(N_{1}\right)$, and [11, Theorem 1] implies that $N_{1}$ is in $U(N)^{-}$. Since $N_{1}$ is not similar to $N$, the proof is complete.

Proposition 2.4. If $N$ is a normal operator on $H$, then $S(N)$ is closed if and only if $\sigma(N)$ is finite.

3. Closure properties of $S(N)$ and $S_{K}(N)$. In this section we consider the closure properties of $S(N)$ and $S_{K}(N)$ for a normal operator $N$ whose spectrum is infinite. We consider first the case when $E(N)$ is finite and obtain a structure theorem for an operator in $S(N)^{-}$.

THEOREM 3.1. Let $N$ be a normal operator in $L(H)$ such that $E(N)$ is finite. If $T$ is in $S(N)^{-}$, then:

(i) there is an invertible operator $S$ and a compact operator $K$ such that $T=S N S^{-1}+K$;

(ii) $\sigma(N)=\sigma(T)$;

(iii) if $z$ is in $\sigma(T)-E(T)$, then $z$ is an isolated eigenvalue of $T$ with finite multiplicity.

Proof. Let $E(N)=\left\{z_{1}, \ldots, z_{n}\right\}$ and let $p(z)=\left(z-z_{1}\right) \ldots\left(z-z_{n}\right)$. The hypothesis implies that $p(T)$ is compact and therefore [10, Theorem 2.4] implies that there is a compact operator $C$ such that $p(T+C)=0$. In particular, we have $E(T)=E(N)$. If $q$ is a polynomial such that $q(T+C)=0$, then $q(E(T))=\{0\}$, and so $q(E(N))=\{0\}$. Then $p$ divides $q$ and is thus the minimal polynomial of $T+C$. As above, there is an invertible operator $S_{1}$ and a diagonable operator $D$ such that $\sigma(D)=E(N)$ and $S_{1} D S_{1}^{-1}=T+C$. Then $E(D)=$ $E(T)=E(N)$ and it follows that there is a unitary operator $U$ and a compact operator $J$ such that $D=U N U^{*}+J$. If $S=S_{1} U$ and $K=S_{1} J S_{1}^{-1}-C$, then we have $T=S N S^{-1}+K$.

(ii) We have $\sigma(N) \subset \sigma(T)$, and since $T$ is polynomially compact, $\sigma(T)$ is countable. Suppose $z$ is in $\sigma(T)-\sigma(N)$. Since $z$ is in $\partial \sigma(T)$, Theorem 3.3 of [3] implies that either $z$ is an isolated point of $\sigma(T)$ and an eigenvalue of finite multiplicity, or $\widetilde{T}-z$ fails to have an inverse in the Calkin algebra. The uppersemicontinuity of the separated parts of the spectrum [15, Theorem 3] implies that the first case cannot arise. However, the second case also cannot arise since $E(T)=E(N)$, and we have a contradiction.

(iii) If $z$ is in $\sigma(T)-E(T)$, then (ii) implies that $z$ is in $\sigma(N)-E(N)$ and is therefore an isolated point of $\sigma(N)=\sigma(T)$. It follows as in (ii) that $z$ is an eigen- 
value of $T$ with finite multiplicity.

LEMma 3.2. If $N$ is a normal operator and $S_{F}(N)=\{X N Y+K: K$ is compact and $\left.\widetilde{X}=\widetilde{Y}^{-1}\right\}$, then $S_{F}(N)=S_{K}(N)$.

Proof. Clearly $S_{K}(N) \subset S_{F}(N)$. To prove the reverse inclusion let $T=$ $X N Y+K$ be an element of $S_{F}(N)$. Now $Y$ is a Fredholm operator and we consider the case index $Y<0$. (Proofs of the other cases are omitted.) There is an operator $L$ and a finite rank operator $G$ such that $L(Y+G)=1$. Let $M$ denote the range of $Y+G$ and let $P$ denote the projection onto $M$. Then $M$ is a subspace of finite codimension in $H$, and the hypothesis implies that $X N Y-$ $L P N(Y+G)$ is compact. Let $N_{M}$ denote the compression of $N$ to $M$ (in $L(M)$ ). Now $L P N(Y+G)$ is similar to $N_{M}$, and the proof is completed by an application of [12, Theorem 2.1 , which implies that $N_{M}$ is unitarily equivalent to a compact perturbation of $N$.

Proposition 3.3. If $N$ is a normal operator on $H$ such that $E(N)$ is finite, then the similarity orbit of $\widetilde{N}$ in the Calkin algebra is closed. If $\widetilde{T}$ is in the closure of the orbit of $\widetilde{N}$, then $T$ is in $S_{K}(N)$.

Proof. Suppose there is an operator $T$ in $L(H)$ and a sequence $\left\{X_{n}\right\}$ of Fredholm operators on $H$ such that $\lim \left\|\widetilde{X}_{n} \widetilde{N} \widetilde{X}_{n}^{-1}-\widetilde{T}\right\|=0$. For each $n$ let $Y_{n}$ denote an operator such that $\widetilde{Y}_{n}=\widetilde{X}_{n}^{-1}$. For each $n,[8, \S 3$, Theorem] implies that there is a compact operator $K_{n}$ such that $\left\|X_{n} N Y_{n}+K_{n}-T\right\|=$ $\left\|\widetilde{X}_{n} \widetilde{N} \widetilde{X}_{n}^{-1}-\widetilde{T}\right\|$. Lemma 3.2 implies that $T$ is in $S_{K}(N)^{-}$. The method of Theorem 3.1 shows that $S_{K}(N)$ is closed and the proof is complete.

The preceding results prove that if $E(N)$ is finite, then $S(N)^{-} \subset S_{K}(N)$, $S_{K}(N)$ is closed, and the similarity orbit of $\widetilde{N}$ is closed in the Calkin algebra. We next prove that if $E(N)$ is infinite, then $S(N)^{-} \not \subset S_{K}(N)$. It follows immediately that $S_{K}(N)$ is not closed and Lemma 3.2 implies that the similarity orbit of $\widetilde{N}$ is not closed in the Calkin algebra.

THEOREM 3.4. If $N$ is a normal operator whose essential spectrum is infinite, then $S(N)^{-} \not \subset S_{K}(N)$. If $M$ is a normal operator whose spectrum is connected and contains $\sigma(N)$, then $M$ is in $S(N)^{-}$.

Proof. We first reduce the proof to the case when $N$ is an essential normal operator. Let $D$ be an essential normal operator such that $\sigma(D)=E(N)$. If the theorem is true for essential normal operators, then $M$ is in $S(D)^{-}$, and therefore $M \oplus N$ is in $S(D \oplus N)^{-}$. Theorem 1 of [11] implies that $D \oplus N$ is in $U(N)^{-}$, and it follows readily that $M \oplus N$ is in $S(N)^{-}$. Since $M$ is an essential normal 
operator, so is $M \oplus N$, and the proof is completed by an application of Theorem B.

Let $N$ be an essential normal operator whose essential spectrum is infinite. Theorem B implies that we may assume $N$ is a diagonable operator each of whose eigenvalues has infinite multiplicity. We may also assume that 0 is a limit point of $E(N)$. Theorem 7 of [7] implies that there is a sequence $\left\{N_{k}\right\}$ of nilpotent operators such that $\lim \left\|N_{k}-M\right\|=0$. Let $i_{k}$ denote the index of nilpotency of $N_{k}$. Theorem 1 of [5] implies that there is an orthogonal decomposition $H=$ $H_{1, k} \oplus \ldots \oplus H_{i_{k}, k}$ with respect to which the operator matrix of $N_{k}$ is in upper triangular form with zeros in each diagonal position. Now each eigenvalue of $N$ has infinite multiplicity; thus, with respect to the decomposition $H \oplus H=$ $H_{1, k} \oplus \ldots \oplus H_{i_{k}, k} \oplus H, N$ is unitarily equivalent to an operator whose matrix is of the form $D_{k}=z_{1, k} \oplus \ldots \oplus z_{i_{k}, k} \oplus N$, where the scalars are distinct and each has modulus less than $1 / k$. Lemma 2.1 implies that there is an invertible operator $S_{k}$ such that $S_{k} N S_{k}^{-1}=N_{k} \oplus 0+D_{k}$. Then we have

$$
\begin{aligned}
\left\|S_{k} N S_{k}^{-1}-M \oplus N\right\| & \leqslant\left\|S_{k} N S_{k}^{-1}-N_{k} \oplus N\right\|+\left\|N_{k} \oplus N-M \oplus N\right\| \\
& <1 / k+\left\|N_{k}-M\right\| .
\end{aligned}
$$

Now $\sigma(N) \subset \sigma(M)$, and Theorem B implies that $M \oplus N$ is in the closure of the unitary orbit of $M$. Since $M \oplus N$ is in $S(N)^{-}$, it therefore follows that $M$ is in $S(N)^{-}$, and the proof is complete.

COROLlARY 3.5. If $N$ is a normal operator whose spectrum is infinite and connected, then a normal operator $M$ is in $S(N)^{-}$if and only if $\sigma(M)$ is connected and contains $\sigma(N)$.

Proof. If $T$ is any operator in $S(N)^{-}$, then [15, Theorem 3] implies that $\sigma(T)$ is connected, and it is also clear that $\sigma(N) \subset \sigma(T)$. The converse follows from the preceding theorem.

Theorem 3.4 implies that if $N$ is normal and $E(N)$ is infinite, then there exist normal operators in $S(N)^{-}-U(N)^{-}$. The next result shows that this cannot occur if $E(N)$ is finite.

COROLlaRY 3.6. If $N$ is normal and $E(N)$ is finite, then each normal operator in $S(N)^{-}$is in $U(N)^{-}$.

Proof. Let $M$ be a normal operator in $S(N)^{-}$. Theorem 3.1 implies that $\sigma(M)=\sigma(N)$ and $E(M)=E(N)$. We will show that if $z$ is an isolated point of $\sigma(N)$, then $\operatorname{dim} \operatorname{ker} N-z=\operatorname{dim} \operatorname{ker} M-z$. It will then follow from Theorem 1 of [11] that $M$ is in $U(N)^{-}$. 
For an operator $T$ such that $\sigma(T)=\sigma(N)$, let $J(T)$ denote the spectral idempotent for $T$ that corresponds to $\{z\}$. Since $N$ and $M$ are normal, $J(N)$ is the projection onto $\operatorname{ker} N-z$ and $J(M)$ is the projection onto $\operatorname{ker} M-z$. Since $M$ is in $S(N)^{-}$, Theorem 3 of [15] implies that there is an invertible operator $S$ such that $J\left(S N S^{-1}\right)$ and $J(M)$ have the same rank. Now $J\left(S N S^{-1}\right)=S J(N) S^{-1}$, and thus $\operatorname{rank} J(N)=\operatorname{rank} J(M)$, which completes the proof.

4. Quasidiagonality and normal operators. Recall that an operator $T$ in $L H$ is quasitriangular (resp., quasidiagonal) if there exists a sequence $\left\{P_{n}\right\}$ of finite rank projections in $L(H)$ such that $P_{n} \stackrel{s}{\rightarrow} 1$ and $\lim \left\|\left(1-P_{n}\right) T P_{n}\right\|=0$ (resp., $\left.\lim \left\|P_{n} T-T P_{n}\right\|=0\right)$ (see [4]). Each normal operator is quasidiagonal, and if $T$ is quasidiagonal, then $T$ and $T^{*}$ are quasitriangular. Each operator similar to a quasitriangular operator is quasitriangular. In [16], R. Smucker proved that a nonquasidiagonal operator may be similar to a quasidiagonal operator.

THEOREM 4.1. A normal operator $N$ in $L(H)$ is similar to a nonquasidiagonal operator if and only if $E(N)$ contains more than two points.

\section{LEMmA 4.2. If $T$ is in $L(H)$ and $T^{2}=T$, then $T$ is quasidiagonal.}

Proof. If $H_{1}$ denotes the range of $T$, then with respect to the decomposition $H=H_{1} \oplus H_{1}^{\perp}, T$ is represented by an operator matrix of the form $\left(\begin{array}{ll}1 & A \\ 0 & 0\end{array}\right)$. We consider first the case $\operatorname{dim} \operatorname{ker} A<\operatorname{dim} \operatorname{ker} A^{*}$ and we let $A=U P$ denote the maximal polar decomposition of $A ; U$ is an isometry and $P \geqslant 0$. The spectral theorem implies that $P$ is the uniform limit of a sequence of invertible diagonable operators, and since the set of all quasidiagonal operators in $L(H)$ is norm-closed, we may assume that $P$ is invertible and diagonable. Since $A^{*} A=P^{2}$, there exists a sequence of finite rank projections $\left\{P_{n}\right\}$ such that $P_{n} \stackrel{s}{\rightarrow} 1$ and such that for each $n, A^{*} A P_{n}=P_{n} A^{*} A$. Let $\left\{e_{k}\right\}$ denote an orthonormal basis for $(A H)^{\perp}$ (if $(A H)^{\perp}$ is $p$-dimensional, set $e_{k}=e_{p}$ for each $k>p$ ). For each integer $n>0$, let $Q_{n}$ denote the projection onto $A P_{n} H \vee\left\langle e_{1}, \ldots, e_{n}\right\rangle$. Since $P$ is invertible, $A$ has closed range and it follows that $Q_{n} \stackrel{s}{\rightarrow} 1$; moreover, an easy calculation shows that for each $n, A P_{n}=Q_{n} A$. If $R_{n}$ denotes the finite rank projection $Q_{n} \oplus P_{n}$, then $R_{n} \stackrel{s}{\rightarrow} 1$, and for each $n, R_{n} T-T R_{n}=0$. This completes the proof of the first case.

We next consider the case $\operatorname{dim} \operatorname{ker} A>\operatorname{dim} \operatorname{ker} A^{*}$. Since quasidiagonality is invariant under adjunction, addition of scalars, and unitary equivalence, it suffices to establish the quasidiagonality of the operator whose matrix is $\left(\begin{array}{cc}1 & -A_{0}^{*} \\ 0\end{array}\right)$, and this follows directly from the preceding case. If $\operatorname{dim} \operatorname{ker} A=\operatorname{dim} \operatorname{ker} A^{*}$, the proof of the first case may be modified by deleting reference to the basis $\left\{e_{k}\right\}$ (since, by the approximation method, we may assume that $A$ is invertible). 
Proof of Theorem 4.1. Theorem 1.2 of [16] implies that if $U$ is a unilateral shift of multiplicity one in $L(H)$, then the operator $T$ on $H \oplus H \oplus H$ whose matrix is

$$
\left(\begin{array}{lll}
0 & 1 & U \\
0 & 0 & 1 \\
0 & 0 & 0
\end{array}\right)
$$

is nonquasidiagonal. It follows from [2, Theorem 8] that $T \oplus 0$ is a nonquasidiagonal operator on $H \oplus H \oplus H \oplus H$. Thus, there exists $\epsilon>0$ such that if $\| S-$ $T \oplus 0 \|<\epsilon$, then $S$ is nonquasidiagonal. Let $N$ be a normal operator whose essential spectrum contains the three distinct numbers $z_{1}, z_{2}$, and $z_{3}$. We may assume $\|N\|<\epsilon$. Theorem A implies that $N$ is unitarily equivalent to a compact perturbation of $z_{1} \oplus z_{2} \oplus z_{3} \oplus N$. Lemma 2.1 implies that the latter operator is similar to $\left(\left(z_{1} \oplus z_{2} \oplus z_{3}\right)+T\right) \oplus N$, which is nonquasidiagonal since $\|N\|<\epsilon$.

For the converse, assume first that $E(N)$ contains exactly one point. Thus $N$ is the sum of a scalar and a compact operator, and the same is true of each operator similar to $N$, which is therefore quasidiagonal. If $E(N)$ contains exactly two points, we may assume $E(N)=\{0,1\}$, so that $N$ is the sum of a projection and a compact operator. Each operator similar to $N$ is therefore the sum of an idempotent and a compact operator, and is therefore quasidiagonal by Lemma 4.2.

We recall that an operator $T$ in $L(H)$ is said to be hyponormal if $T^{*} T-$ $T T^{*} \geqslant 0 ; T$ is said to be seminormal if either $T$ or $T^{*}$ is hyponormal (see [11]).

COROLlARY 4.3. A seminormal operator $T$ in $L(H)$ is similar to a nonquasidiagonal operator if and only if $E(T)$ contains more than two points.

Proof. Since the cardinality of $E(T)$ is equal to that of $E\left(T^{*}\right)$, and since an operator is quasidiagonal if and only if its adjoint is quasidiagonal, we may assume that $T$ is hyponormal. If $E(T)$ contains one or two points, the result will follow from Theorem 4.1 once we prove that in this case $T$ is normal. Indeed, since $E(T)$ is finite, Theorem 1 of [13] implies that $\sigma(T)$ is countable, and since $T$ is hyponormal, Theorem 1 of [14] now implies that $T$ is normal. For the converse, using the notation of the proof of Theorem 4.1, it suffices to prove that $z_{1} \oplus z_{2} \oplus z_{3} \oplus T$ is unitarily equivalent to a compact perturbation of $T$, and this fact is a direct consequence of [11, Theorem 1].

ADDED IN PROOF. We have received from Professor Herrero a preprint of his paper Closure of similarity orbits of Hilbert space operators. III and preprints of several earlier papers on related subjects. Among the diverse results of these papers, Professor Herrero has independently obtained numerous interesting extensions of the main results of $\S 3$ (Theorems 3.1 and 3.4). 


\section{REFERENCES}

1. L. Brown, R. Douglas and P. Fillmore, Unitary equivalence modulo the compact operators and extensions of $C^{*}$ algebras, Springer Notes Series, 1973.

2. R. G. Douglas and Carl Pearcy, A note on quasitriangular operators, Duke Math. J. 37 (1970), 177-188. MR 41 \#2439.

3. P. A. Fillmore, J. G. Stampfli and J. P. Williams, On the essential numerical range, the essential spectrum, and a problem of Halmos, Acta Sci. Math. (Szeged) 33 (1972), 177192. MR 48 \#896.

4. P. R. Halmos, Ten problems in Hilbert space, Bull. Amer. Math. Soc. 76 (1970), 887-933. MR 42 \#5066.

5. - Capacity in Banach algebras, Indiana Univ. Math. J. 20 (1970/71), 855863. MR 42 \#3569.

6. - Limits of shifts, Acta Sci. Math. (Szeged) 34 (1973), 131-139.

7. D. A. Herrero, Normal limits of nilpotent operators, Indiana Univ. Math. J. 23 (1974), 1097-1108.

8. R. B. Holmes and B. R. Kripke, Best approximation by compact operators, Indiana Univ. Math. J. 21 (1971), 255-263. MR 45 \#5718.

9. T. Hoover, Thesis, University of Michigan, Ann. Arbor, Mich., 1970.

10. C. L. Olsen, A structure theorem for polynomially compact operators, Amer. J. Math. 93 (1971), 686-698.

11. C. Pearcy and N. Salinas, Compact perturbations of seminormal operators, Indiana Univ. Math. J. 22 (1973), 789-793. MR 47 \#2404. 115-120.

12. - Operators with compact self-commutator, Canad. J. Math. 26 (1974),

13. C. R. Putnam, The spectra of operators having resolvents of first-order growth, Trans. Amer. Math. Soc. 133 (1968), 505-510. MR 37 \#651.

14. - An inequality for the area of hyponormal spectra, Math. Z. 116 (1970), 323-330. MR 42 \#5085.

15. N. Salinas, Operators with essentially disconnected spectrum, Acta Sci. Math. (Szeged) 33 (1972), 193-205.

16. R. Smucker, Quasidiagonal and quasitriangular operators, Thesis, Indiana University, Bloomington, Ind., 1973.

DEPARTMENT OF MATHEMATICS, WESTERN MICHIGAN UNIVERSITY, KALAMAZOO, MICHIGAN 49001 